\title{
Rate of presentation and the acoustic similarity effect in short-term memory
}

R. CONRAD, A, D. BADDELEY AND A, J. HULL

MEDICAL RESEARCH COUNCIL, APPLIED PSYCHOLOGY RESEARCH UNIT, CAMBRIDGE, ENGLAND

Ss attempted to recall sequences of six consonants drawn from either an acoustically similar set (B C D G P Q T V), or from a relatively dissimilar set ( $H K M P R S W Y$ ). Letters were presented visually at a rate of 60 or 120 letters per min. Performance was impaired by acoustic similarity $(p<.001)$ but there was no effect of rate of presentation and no interaction between rate and similarity. This does not support a limited channel capacity interpretation of the acoustic similarity effect.

Sequences of items which are hard to discriminate when heard in noise are also difficult to retain in short-term memory (STM), even though presented visually. This effect is large and holds for both consonants (Conrad, 1964) and words (Conrad, 1963), where its effects are considerably greater than the equivalent effects of semantic similarity (Baddeley, in press).

This phenomenon is open to a wide range of possible explanations including intra-sequence interference (Wickelgren, 1965a) and decay of discriminative cues (Conrad, 1964). A further class of possible explanations, however, might be in terms of the overloading of a limited informational channel. There is ample evidence that the STM system has a limited capacity (Broadbent, 1958; Posner \& Rossman, 1965; Murdock, 1965), and it seems possible that acoustically similar material overloads the system at some point. If this is the case a faster rate of presentation might be expected to interact with acoustic similarity and by stressing an already overloaded system, to produce an even lower level of performance on the acoustically similar material. The following experiment tests this prediction. Method

Twenty-four six-letter sequencies were constructed from each of two vocabularies, one in which acoustic similarity was high (B C DG PQ T V; mean probability of acoustic confusion $=.73$ ) (Conrad \& Hull, 1964), and one in which similarity was relatively low (H K M P R S W Y; mean probability of confusion $=.18$ ). The sequences were constructed with two major constraints: (1) All letters occurred equally often in each serial position. (2) The sets were balanced for the number of sequences having 0,1 or 2 letters repeated (Wickelgren, 1965b).

Sequences were filmed one letter per frame $(16 \mathrm{~mm})$ with warning symbols preceding each sequence. Presentation was by single frame projection at a rate of either 60 or 120 frames $/ \mathrm{min}$. Ss, 24 enlisted men, were
Table 1. Effect of Presentation Rate and Acoustic Similarity on Recall of Sequences. (Percent wrong sequences)

\begin{tabular}{lcc} 
& Similar & Dissimilar \\
\hline Fast Rate & 88 & 39 \\
Slow Rate & 82 & 34 \\
\hline
\end{tabular}

tested individually. They were given each set of material twice, once at each speed. They were thus tested on one condition on each of four successive days with the order of test counterbalanced. The Ss were instructed to write down their Rs in order of presentation; they were encouraged to guess rather than omit Rs, and to maximize $\mathbf{R}$ availability the appropriate vocabulary was permanently displayed. A sequence was presented as soon as $\mathbf{R}$ to the previous sequence was complete. Results

Table 1 shows percent incorrect sequences for the four conditions. Analysis of variance shows the effect of acoustic similarity to be highly significant ( $F=234.6$; $\mathrm{df}=1 / 66 ; \mathrm{p}<.001)$, while neither the effect of rate $(F=3.30 ; \mathrm{df}=1 / 66 ; p>.05)$ nor the interaction $(F=0.96$; $\mathrm{df}=1 / 66 ; \mathrm{p}>.05$ ) was significant.

\section{Discussion}

It is clear that increasing rate of presentation does not differentially impair recall of acoustically similar sequencies. While this does not of course eliminate all channel capacity interpretations of the acoustic similarity effect, it does limit the range of possible models quite considerably. More specifically it suggests that at a $60 / \mathrm{min}$. rate $S$ has ample time to perceive and encode the incoming material since doubling the rate does not impair performance. This does not of course mean that encoding is therefore optimal, but it does imply that any encoding difficulty is not due to the rate at which the encoding system can process information. The possibility remains, however, that the acoustic similarity effect may be due to a limited capacity retrieval mechanism, since $R$ time was not varied across conditions. However, since responding was unpaced, it seems implausible to assume that the retrieval mechanism was overloaded, and while it is possible that acoustic similarity leads to slower retrieval which in turn leads to more forgetting, Wickelgren's (1966) demonstration of an equivalent acoustic similarity effect in a delayed recognition situation makes this seem unlikely. It therefore seems more probable that the acoustic similarity effect in STM is attributable to the loss of crucial discriminative cues. 


\section{References}

Baddeley, A. D. Short-term memory for word sequences as a function of acoustic semantic and formal similarity. Quart. $J$. exp Psychol., in press.

Broadbent, D. E. Perception and communication. New York: Per gamon Press, 1958.

Conrad, R. Acoustic confusion and memory span for words. Nature, $1963,197,1029-1030$.

Conrad, R. Acoustic confusion and immediate memory. Brit. $J$ Psychol., 1964, 55, 75-84.

Conrad, R., \& Hull, A. J. Information, acoustic confusion and memory span. Brit. J. Psychol., 1964, 55, 429-432.
Murdock, B. B. Effects of a subsidiary task on short-term memory. Brit. J. Psychol., 1965, 56, 413-420.

Posner, M. I., \& Rossman, E. Effect of size and location of informational transform upon short-term retention. J.exp. Psychol. $1965,70,496-505$.

Wickelgren, W. A. Short-term memory for phonemically similar lists. Amer. J. Psychol, 1965a, 78, 567-574.

Wickelgren, W. A. Short-term memory for repeated and non-repeated items. Quart, J. exp. Psychol., 1965b, 17, 14-25.

Wickelgren, W. A. Short-term recognition memory for single letters and phonemic similarity of retro-active interference, Quart. J. exp. Psychol., 1966, 18, 55-63. 